\title{
Sehr geehrte Leserinnen und Leser!
}

\author{
${ }^{1} \mathrm{Graz}$, Österreich \\ ${ }^{2}$ Leoben, Österreich
}

Christof Sommitsch ${ }^{1}$, Bruno Hribernik ${ }^{2}$

Online publiziert am 13. August 2015

Die 8. European Stainless Steel Conference 2015 (ESSC) und die Duplex Steel Conference \& Exhibition 2015 fanden vom 28. bis 30. April 2015 in Graz statt. Ein anspruchsvolles Programm mit mehr als 85 Fachvorträgen rund um das Thema der Produktionstechnik von rostfreien Stählen, wie etwa Warm- und Kaltwalzen, Wärmebehandlung und vieles mehr, erwartete die rund 200 internationalen Experten aus Wissenschaft und Industrie.

Die International Society of Steel Institutes (ISSI) hat diese Konferenz zum ersten Mal nach Österreich an die ASMET - The Austrian Society for Metallurgy and Materials - vergeben. Die Tagung brachte Spezialisten aus Wissenschaft und Industrie aus 24 Nationen zusammen, um die neuesten technologischen Innovationen zu präsentieren und zu diskutieren. Es wurden die aktuellsten Ergebnisse aus den Bereichen Werkstoffentwicklung, Fortschritte in der Produktionstechnologie (Stahlherstellung, Warm- und Kaltwalzen, Wärmebehandlung, Verarbeitung etc.), Anlagenbau, Korrosionsverhalten, Verarbeitung und Anwendungsmöglichkeiten aus rostfreiem Stahl vorgestellt. Einen weiteren wesentlichen Schwerpunkt der Konferenzen stellten die Fügtechnik und das Schweißen von rostfreien Stählen dar.

Möglichkeiten zum Austausch unter Experten über Entwicklungen zu bestehenden und potenziellen Anwendungen und über marktrelevante Faktoren rundeten die Veranstaltungen ab.

Die Tagung zielte darauf ab, die Wettbewerbsfähigkeit der europäischen Hersteller von rostfreien Stählen zu steigern und auch die Verwendung von rostfreien Stählen zu fördern. Die Konferenz sah sich auch als Impulsgeber für neue Anwendungen und als Hilfe für die zukünftigen Werkstoffentwicklungen am Rostfreisektor. Aktuelle Trends in der Entwicklung der Märkte und Anwendungen von rostfreien Stählen und insbesondere der Duplex Stähle prä-

sentierte Markus Moll, Geschäftsführer und Senior Market Analyst bei SMR - Steel \& Metals Market Research. Die Paneldiskussion mit Vertretern namhafter Hersteller rostfreier Stähle und Metallurgie-Anlagen sowie mit Öl- und Gasexperten beleuchtete die zukünftige Entwicklung speziell im Öl- und Gas-Segment.

Die sehr gute Organisation und der reibungslose Ablauf der Konferenz war dem professionellen Eventmanagement der ASMET und dem Institut für Werkstoffkunde und Schweißtechnik an der Technischen Universität Graz zu verdanken. Namentlich möchten wir der Conference Administration danken: Fr. Melanie Baumgartner - Austrian Society for Metallurgy and Materials - und Fr. Isabella Scheiber - Technische Universität Graz.

Die für dieses Heft ausgewählten Beiträge der ESSC2015C zeigen einen hochinteressanten Querschnitt aus den wichtigsten Themenbereichen der Konferenz.

Wir wünschen viele interessante und neue Erkenntnisse beim Lesen der ausgewählten Konferenzbeiträge.

Christof Sommitsch Scientific Chairman

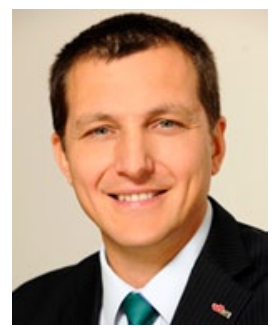

Bruno Hribernik Organization Chairman

Christof Sommitsch Graz, Österreich

E-Mail: christof.sommitsch @ tugraz.at 